\title{
A novel variable M/T method for speed measurement with high precision in a wide speed range
}

\author{
Jian-tao $\mathrm{Pu}^{1, \mathrm{a}}$, Hui Wang ${ }^{2,3, \mathrm{~b}}$ \\ ${ }^{1}$ College of Automation, Beijing Union University, Beijing, China \\ ${ }^{2}$ College of Automation, Beijing Information Science \& Technology University, Beijing, China \\ ${ }^{3}$ Institute of Automation, Chinese Academy of Sciences, Beijing, China \\ apu.jiantao@hotmail.com, bwanghui02@tsinghua.org.cn
}

Keywords: Optical incremental encoder, leap variable M/T method, speed measurement

\begin{abstract}
In this paper, a new variable $\mathrm{M} / \mathrm{T}$ method called leap variable $\mathrm{M} / \mathrm{T}$ method is introduced for speed measurements by optical incremental encoders in motor servo systems. In this method, both encoder pulse and high frequency clock pulse are counted in a variable interval as conventional variable $\mathrm{M} / \mathrm{T}$ method which ensures the high measurement accuracy in both high speed and low speed. Furthermore by leap trigger time of counting period, the speed delay can be reduced by at least one sampling period, which guarantees a more accurate speed feedback signal and rapid response of motor system.
\end{abstract}

\section{Introduction}

A rotary encoder, also called a shaft encoder, is an electro-mechanical device that converts the angular position or motion of a shaft to an analog or digital code. The optical incremental encoder is the most widely used of all rotary encoders due to its low cost and ability to provide digital signals that can be easily interpreted to provide motion related information such as velocity. They are used as speed sensors in many applications that require precise shaft unlimited rotation including industrial controls, robotics and so on.

Optical incremental encoder has slits at the circumference and produces pulse train as it rotates. The speed is evaluated from the pulse train coming from the encoder. Previous studies have shown many different ways to measure the speed. Counting the pulse train with fixed sampling interval called M method. However M method is more applicable at medium and high speeds rather than at low speed, since at low speed the pulses are not frequently produced and no pulse will be detected in some sampling periods[1-4]. An alternative way is to measure the time interval between two successive pulses, which called T method. The approach has high resolution at low speed but has unacceptable error at high speed[1-4]. Obviously in wide speed range neither $\mathrm{M}$ method nor $\mathrm{T}$ method is suitable. Combination of these methods is proposed by Ohmae et al in [1], called M/T method. This method is to measure pulse train and clock pulses at a constant detecting time Td so that it combines the advantages of $\mathrm{M}$ and $\mathrm{T}$ methods with high accuracy in wide speed range. In reference [4-6], the constant elapsed time (CET) method is proposed which measures the elapsed time between $\mathrm{k}$ successive pulses, and dynamically adjusts the value of $\mathrm{k}$ to obtain a near constant response time. E.Galvan et al improve the aforementioned method by several adaptive techniques to maintain the accuracy of the results while preserving the short system response time[7-10].

All of these methods are solutions to solve the speed estimate in wide speed span with high accuracy. They still can not solve the problem of long time delay even though the resolution is raised. In this paper, a new approach of speed measurement with low resolution optical incremental encoder is proposed by shorter delay for high accuracy in wide speed range. This approach is based on variable $\mathrm{M} / \mathrm{T}$ method, but has a leap counting trigger time which will reduce the time delay for at least one sample period with respect to variable $\mathrm{M} / \mathrm{T}$ method. Therefore the accuracy maintains high in wide speed range with rapid response at the same time. It is particularly suitable for the advanced 
numerical control system or servo system in industry robots which have strictly rapid response and wide speed range requirements.

This paper is organized as follows: a new speed measurement method is introduced in Section II. An implement is discussed in details in Section III, while Section IV gives the final conclusions.

\section{Methods}

M/T method. This method measures not only encoder pulse $\mathrm{m} 1$ but also the clock pulse $\mathrm{m} 2 \mathrm{in}$ fixed counting period (usually the system sampling period Ts), as shown in Fig.1.

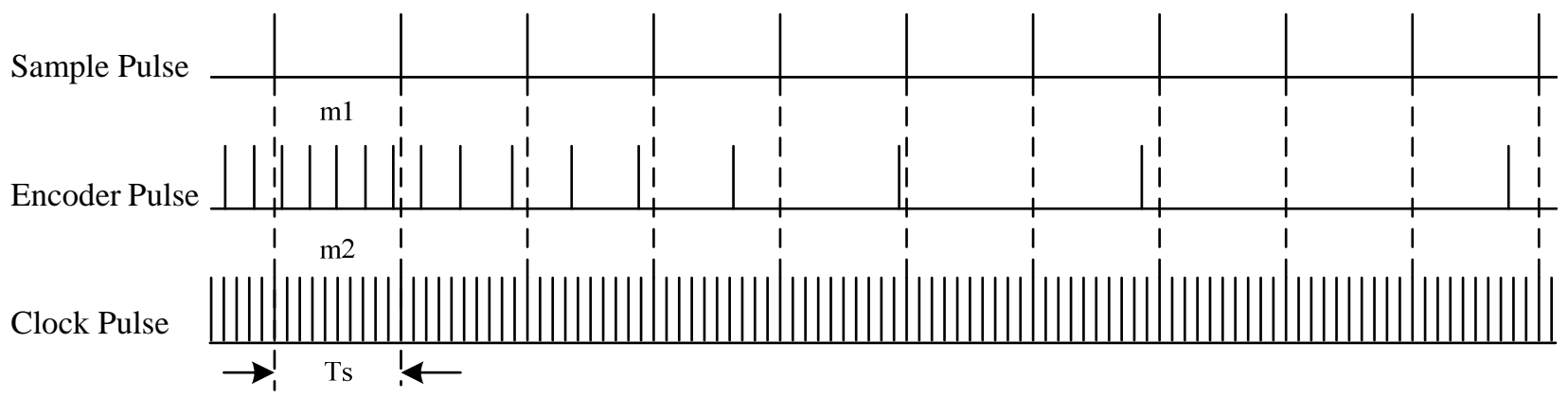

Figure 1. M/T method

As known the clock pulse frequency $f_{0}$ and the number of slits at the encoder circumference $\mathrm{Z}$, speed can be given by Eq.1.

$$
n=\frac{60 f_{0} m_{1}}{Z m_{2}} \text {. }
$$

$\mathrm{M} / \mathrm{T}$ method improves the accuracy greatly at low speed and can be easily implemented by QEP unit of DSP. However as the M method, it still has the problem of low accuracy at very low speed when m1(the amount of encoder pulses in one sampling period) will decrease to be zero in some discontinuous sampling periods as shown in Fig.1. As a result the system performance will deteriorate by intermittent speed feedback. To overcome this shortcoming, the variable M/T method comes into being.

Variable M/T method. The difference between M/T method and variable M/T method lies in the counting time. Variable $\mathrm{M} / \mathrm{T}$ method has variable counting intervals. For variable M/T method, counting time begins at the first rising edge of the encoder pulse in sampling period and finishes at the end of a whole encoder cycle in the current sampling period, as shown in Fig.2.

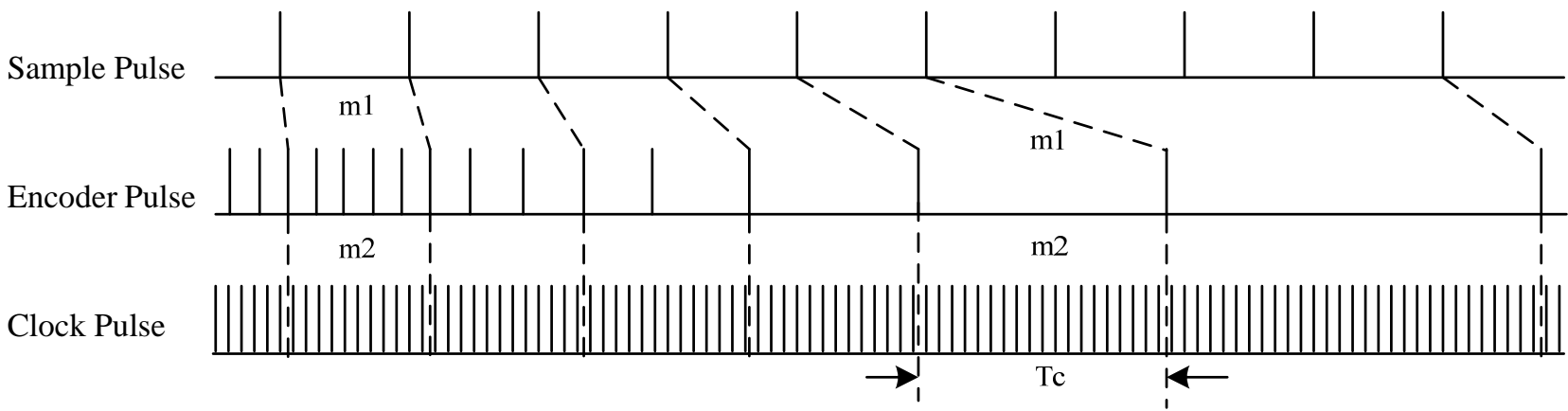

Figure 2. Variable M/T method

In this method, the motor speed is given by the same equation as Eq.1 of the M/T method. Attention to the trigger time of counting time Tc delaying the sampling time Ts at the rising edge of the encoder pulse following the current sampling period. This can bring two advantages. First, the number $\mathrm{m} 2$ is counted in the period of $\mathrm{m} 1$ complete encoder cycles. The error will be one clock pulse which is much shorter than encoder pulse. It can decrease the counting error effectively. Second, it unifies M method and T method in one method. At high speed shown in the left part of Fig.2., encoder frequency is larger than sampling frequency, Tc approximates Ts. In this case, variable M/T method 
has the same effect with $\mathrm{M} / \mathrm{T}$ method and the relative error is nearly the same. As speed decreasing to a very low value that encoder cycle is much longer than sampling period as shown in the right part of Fig.2, variable $M / T$ method turns to be $T$ method when $\mathrm{m} 1$ equals to one. At that time variable $\mathrm{M} / \mathrm{T}$ method has the same relative error with $\mathrm{T}$ method. Concisely variable $\mathrm{M} / \mathrm{T}$ method can gain a high accuracy in quite wide speed range. Actually the measured speed is an average velocity during the counting time and can be regarded approximately as the actual speed at the middle of the counting time. But the principle of the variable $\mathrm{M} / \mathrm{T}$ method causes the delay of speed value sampling by nearly one sampling period as shown in Fig.2. The delay of the measurement may be unacceptable in the applications required rapid response.

Leap variable $\mathbf{M} / \mathbf{T}$ method. To improve the variable $\mathrm{M} / \mathrm{T}$ method, the counting time should begin at the previous encoder pulse before the following sampling period as shown in Fig.3. When the sampling pulse comes, the counters have finished counting the value of $\mathrm{m} 1$ and $\mathrm{m} 2$ already. The delay of speed measurement can be decreased by nearly one sampling period Ts. The change of the counting trigger time has no effect on the speed calculation and the speed value is given by the same equation as Eq.1 of the M/T method and the variable M/T method. This method has the same resolution as the variable $\mathrm{M} / \mathrm{T}$ method.

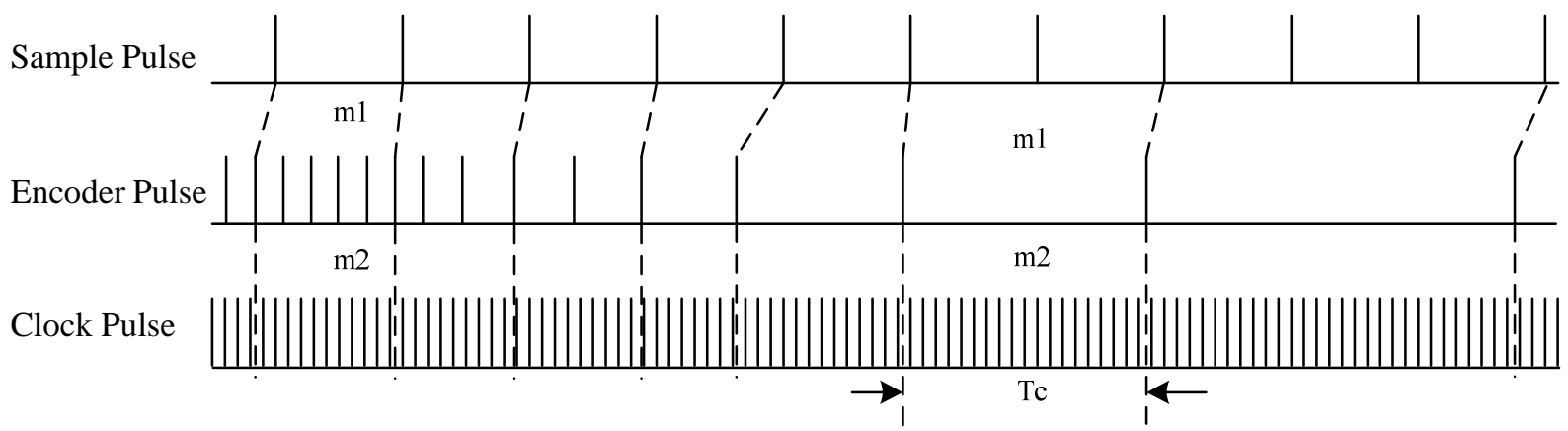

Figure 3. Leap Variable M/T method

\section{Discussion}

The leap variable $\mathrm{M} / \mathrm{T}$ method has an obvious advantage of less delay than other methods. But it is more difficult to implement because it can not to predict the exact time of the last encoder pulse to the next sample pulse. Variable $\mathrm{M} / \mathrm{T}$ method can be implemented easily by interruptions of the sample pulse and encoder pulse. While the leap variable M/T method can not be done by the same way. Fortunately CPLD can be used to implement the algorithm rather than by exterior interruptions of DSP or other microcontrollers. Two Counters for encoder pulse and clock pulse and several latches were realized in CPLD. At the rising edge of every encoder pulse, counter value of the clock pulse latched in a register. Then at the following rising edge of sample pulse, $\mathrm{m} 1$ was gotten by the difference between the latched encoder pulse counter value and the previous latched encoder pulse value in the last sample period and $\mathrm{m} 2$ can be gained by subtracting the latched clock pulse counting value from the last clock pulse value of last sample period. By this way the speed can be calculated from $\mathrm{m} 1$ and $\mathrm{m} 2$ by Eq.1.

\section{Summary}

In this paper, a novel incremental encoder-based motor speed measurement has been proposed. It is based on variable $\mathrm{M} / \mathrm{T}$ method which provides an alternative counting period between the system sampling period at high speed and encoder cycle at low speed, so that it can gain high accuracy for a wide speed range. A leap counting trigger time makes the improved method has less delay (ahead at least one sample period) than the conventional variable $\mathrm{M} / \mathrm{T}$ method. It will offer a more accurate speed feedback signal for the following speed estimator or speed controller and improve the performance in a wide speed range. For the advanced numerical control system or servo system in 
industry robots which have strictly rapid response and wide speed range requirements, the leap variable $\mathrm{M} / \mathrm{T}$ method is particularly suitable.

\section{References}

[1] T. Ohmae, T. Matsuda, K. Kamiyama, M. Tachikawa, in:A microprocessor-controlled high-accuracy wide-range speed regulator for motor drives, IEEE Trans. Ind. Electron., vol. IE-29, no. 3, p. 207-221 ( 1982)

[2] Lilit Kovudhikulrungsri,Takafumi Koseki. in:Precise speed estimation from a low-resolution encoder by dual-sampling-rate observer, IEEE/ASME Transactions on Mechatronics, vol.11, no. 6, p.661-670 (2006)

[3] Ndubuisi Ekekwe, Ralph Etienne-Cummings, Peter Kazanzides, in: A wide speed range and high precision position and velocity measurements chip with serial peripheral interface, Integration, the VLSI Journal, vol. 41, no. 2, p.297-305 (2008)

[4] Richard Bonert, in: Digital Tachometer with Fast Dynamic Response Implemented by a Microprocessor, IEEE Transactions on Industry Applications, vol.IA-19, no.6, p.1052-1056(1983)

[5] Shih-Chin Yang, Robert D. Lorenz, in:Surface Permanent-Magnet Machine Self-Sensing at Zero and Low Speeds Using Improved Observer for Position, Velocity, and Disturbance Torque Estimation, Transactions on Industry Applications, vol.48, no.1,p.151-160(2012)

[6] Richard Bonert, in: Design of a high performance digital tachometer with a microcontroller, IEEE Transactions on Instrumentation and Measurement. vol.38, no.6, p. 1104-1108(1989)

[7] N.Hagiwara, Y.Suzuki, H.Murase, in: A method of improving the resolution and accuracy of rotary encoders using a code compensation technique, IEEE Transactions on Instrumentation and Measurement. vol.41, no.1, pp.98-101(1992)

[8] E.Galvan, A.Torralba, L.G.Franquelo, in: A simple digital tachometer with high precision in a wide speed range, Industrial Electronics, Control and Instrumentation, vol.2, p. 920-923(1994)

[9] E.Galvan,A.Torralba,L.G.Franquelo,in: ASIC implementation of a digital tachometer with high precision in a wide speed range, IEEE Transactions on Industrial Electronics,vol.43, no. 6, p. 655 660(1996)

[10] P. Bhatti, B. Hannaford, in: Single-chip velocity measurement system for incremental optical encoders, IEEE Transactions on Control System Technology, vol.5, no.6(1997) 